\title{
CONVEX DOMAINS AND LINEAR COMBINATIONS OF CONTINUOUS FUNCTIONS*
}

BY I. J. SCHOENBERG

1. Introduction. Dines' Problem. Let there be given $n$ real, continuous and linearly independent functions

$$
\phi_{1}(x), \phi_{2}(x), \cdots, \phi_{n}(x), \quad(a \leqq x \leqq b),
$$

which we may interpret as cartesian coordinates of an arc of curve $\Gamma$ in the $n$-space $S_{n}$. Let $K=K(\Gamma)$ denote the smallest closed convex domain in $S_{n}$ containing the arc $\Gamma$. The following lemma is due to F. Riesz. $†$

Lemma 1. $A$ point $X=\left(x_{1}, x_{2}, \cdots, x_{n}\right)$ belongs to $K$ if and only if its coordinates can be represented in the form

$$
x_{k}=\int_{a}^{b} \phi_{k}(x) d \psi(x), \quad(k=1,2, \cdots, n),
$$

where $\psi(x)$ is monotonic and non-decreasing in $[a, b]$, with $\psi(a)=0, \psi(b)=1$.

A characterization of the points which are interior to $K$ is given by the following lemma.

Lemma 2. $A$ point $X=(x)$ is an interior point of $K$ if and only if its coordinates can be represented in the form

$$
x_{k}=\int_{a}^{b} p(x) \phi_{k}(x) d x, \quad(k=1,2, \cdots, n),
$$

where $p(x)$ is continuous $\ddagger$ and positive in $[a, b]$ and $\int_{a}^{b} p(x) d x=1$.

This lemma is proved in $\$ 4$ below. I wish to show now how by

* Presented to the Society, April 14, 1933.

$\dagger$ †. Riesz, Annales de l'École Normale, (3), vol. 28, pp. 56-57. It can be derived immediately from some fundamental properties of convex domains to be found in Chapter I of C. Carathéodory's article Über den Variabilitätsbereich der Fourierschen Konstanten von positiven harmonischen Funktionen, Rendiconti di Palermo, vol. 32 (1911), pp. 193-217. The reader is referred to this place for all the information about convex domains which is used in this note.

$\ddagger$ Concerning this assumption on $p(x)$ see the concluding remark at the end of this note. 
means of these lemmas some interesting questions about linear combinations of the functions (1) can be readily answered. We start with the following problem stated and solved by L. L. Dines.*

Under what conditions does every real linear combination

(4) $\Phi(x)=a_{1} \phi_{1}(x)+a_{2} \phi_{2}(x)+\cdots+a_{n} \phi_{n}(x), \quad\left(\sum_{1}^{n} a_{k}^{2}>0\right)$, change sign in the interval $[a, b]$ ?

That this should be so, it is obviously necessary and sufficient that every hyperplane

$$
\pi(x) \equiv a_{1} x_{1}+a_{2} x_{2}+\cdots+a_{n} x_{n}=0, \quad\left(\sum_{1}^{n} a_{k}^{2}>0\right)
$$

passing through the origin $O$ of $S_{n}$ shall cut the $\operatorname{arc} \Gamma$, that is, that the origin $O=(0,0, \cdots, 0)$ be an interior point of $K$. From this remark and Lemma 2, we immediately derive the following answer.

Theorem 1 (L. L. Dines). Every linear combination (4) will change sign in $[a, b]$ if and only if there exists a positive continuous function $p(x)$ with

$$
\int_{a}^{b} p(x) \phi_{k}(x) d x=0, \quad(k=1,2, \cdots, n) .
$$

By similar reasons every $\Phi(x)$ will vanish somewhere in $[a, b]$ if and only if the origin $O$ is a point of $K$. Hence Lemma 1 gives the following result.

THEOREM 2. Every linear combination (4) will vanish somewhere in $[a, b]$ if and only if there exists a monotonic function $\psi(x)$ not identically constant in $[a, b]$ with

$$
\int_{a}^{b} \phi_{k}(x) d \psi_{k}(x)=0, \quad(k=1,2, \cdots, n) .
$$

* L. L. Dines, $A$ theorem on orthogonal functions with an application to integral inequalities, Transactions of this Society, vol. 30 (1928), pp. 425-438. See also L. L. Dines, Annals of Mathematics, (2), vol. 28 (1926), pp. 393-395, and N. H. McCoy, this Bulletin, vol. 36 (1930), pp. 878-882.

$\dagger$ That is to say, have points of the arc $\Gamma$ on both of its sides. 
2. Generalization of Dines' Problem. A recent article of J. Favard* suggests the following generalization of the problem of Dines: For what constants $c_{1}, c_{2}, \cdots, c_{n},\left(\Sigma c_{k}^{2}>0\right)$, does every linear combination (4), whose coefficients satisfy the relation

$$
a_{1} c_{1}+a_{2} c_{2}+\cdots+a_{n} c_{n}=0,
$$

change sign in $[a, b]$ ?

An answer to this question is given in the following theorem.

TheOREм 3. Every linear combination (4), whose coefficients satisfy the relation (6), changes sign in $[a, b]$ if and only if the system of equations

$$
\int_{a}^{b} p(x) \phi_{k}(x) d x=c_{k}, \quad(k=1,2, \cdots, n),
$$

has a continuous solution $p(x) \neq 0$ in $[a, b] . \dagger$

The proof of this theorem requires the following additional geometric lemma whose proof we postpone to the end of this note $(\$ 5)$.

LEмма 3. (a) If a straight line $\Delta$ of $S_{n}$ has no point in common with $K$, then we can pass through $\Delta$ a hyperplane $\pi$ which also has no point in common with $K$, that is, $\pi$ is a bound of $K$. (b) If $\Delta$ and $K$ have points in common none of which is interior to $K$ (we may call $\Delta$ a line of support of $K$ ), then we can pass through $\Delta a$ hyperplane of support $\pi$ of $K$.

Let us return to the proof of Theorem 3. Let $\Delta$ denote the straight line joining the point $C=\left(c_{1}, c_{2}, \cdots, c_{n}\right)$ to the origin $O$. Lemma 3 gives the following geometric criterion: Every $\Phi(x)$, satisfying (6), will change sign in $[a, b]$ if and only if $\Delta$ contains an interior point $P$ of $K$. Indeed, if $\Delta$ contains such a point $P$, then every hyperplane (5) through $\Delta$ will cut the arc $\Gamma$, i.e., every $\Phi(x)$ satisfying (6) will change sign in $[a, b]$. Conversely, if $\Delta$ contains no point interior to $K$, then Lemma 3 shows that there exists a linear combination $\Phi(x)$, satisfying (6), which

* J. Favard, Sur les zéros réels des polynômes, Bulletin de la Société Mathématique, vol. 59 (1931), pp. 229-255.

$\dagger$ If every $\Phi(x)$ changes sign in $[a, b]$, irrespective of (6), then system (7) has a required solution $p(x)$ for any constants $c_{1}, c_{2}, \cdots, c_{n}$. Indeed, the origin $O$ is then interior to $K$. 
does not change sign in $[a, b]$. Moreover, if $\Delta$ contains interior points of $K$, it will also contain such a point $P \neq O$. The coordinates of $P$ expressed in the form (3) and multiplied by the ratio $\overline{O C} / \overline{O P}(\neq 0)$, yield the characteristic representation (7) of the coordinates of $C$. Similarly, Theorem 2 is generalized by the following theorem.

THEOREM 4. We assume that not every linear combination (4) vanishes somewhere in $[a, b]$. Under this assumption every $\Phi(x)$, whose coefficients satisfy (6), will vanish somewhere in $[a, b]$ if and only if the system

$$
\int_{a}^{b} \phi_{k}(x) d \psi(x)=c_{k}, \quad(k=1,2, \cdots, n),
$$

has a monotonic solution $\psi(x)$ not identically constant in $[a, b]$.

Indeed, the first assumption of this theorem means that $O$ is an exterior point of $K$. Then relation (6) implies that $\Phi(x)$ vanishes in $[a, b]$ if and only if the straight line $\Delta$ passing through $O$ and $C$ has at least one point $P$ in common with $K$ (Lemma 3, case (a)). The coordinates of $P$ expressed in the form (2), and multiplied by the ratio $\overline{O C} / \overline{O P}(\neq 0)$, yield the characteristic representation (8) of the coordinates of $C$. It is easy to see geometrically that Theorem 4 fails to be always true if we drop the assumption of its first sentence.

3. Infinite Sequences of Functions. Let there be given an infinite sequence of linearly independent continuous functions

$$
\phi_{0}(x) \equiv 1, \phi_{1}(x), \phi_{2}(x), \cdots, \quad(a \leqq x \leqq b),
$$

and let

$$
c_{0}>0, c_{1}, c_{2}, \cdots,
$$

be a given sequence of real constants. Again it is suggested by Favard's paper that we ask the following question. For what sequences (10) does, for every $n$, a linear combination

$$
\Phi(x)=a_{0} \phi_{0}(x)+a_{1} \phi_{1}(x)+\cdots+a_{n} \phi_{n}(x),
$$

whose coefficients satisfy the relation

$$
a_{0} c_{0}+a_{1} c_{1}+\cdots+a_{n} c_{n}=0,
$$

always vanish, at least once, in $[a, b]$ ? 
We shall prove the following theorem.

THEOREM 5. For every n, a linear combination (11), whose coefficients satisfy (12), will always vanish at least once in $[a, b]$ if and only if the system

$$
\int_{a}^{b} \phi_{k}(x) d \psi(x)=c_{k}, \quad(k=0,1,2, \cdots)
$$

admits a monotonic solution $\psi(x)$.

The condition of this theorem is obviously sufficient. Its necessity is readily derived from Theorem 4 and a well known theorem of Helly.*

Indeed, on account of Theorem 4, the finite system

$$
\int_{a}^{b} \phi_{k}(x) d \psi_{n}(x)=c_{k}, \quad(k=0,1,2, \cdots, n),
$$

admits, for every $n$, a monotonic solution $\psi_{n}(x)$, with $\psi_{n}(a)=0$. By Helly's theorem there exists a subsequence $\psi_{n_{i}}(x)$ which converges to a monotonic solution $\psi(x)$ of the system (13).

For the special sequence of functions

$$
\phi_{0}(x)=1, \phi_{1}(x)=x, \phi_{2}(x)=x^{2}, \cdots,
$$

Theorem 5 was given by Favard (loc. cit. p. 244). For this particular sequence (14), Favard proved Theorem 5 also when one or both of the limits $a$ and $b$ are infinite. Our method can not cover these cases, since Theorem 4 , on which it is based, fails to be true if $a=-\infty, b=+\infty, n \geqq 3$, for example, if $c_{0}=1$, $c_{1}=c_{2}=\cdots=c_{n-1}=0, c_{n}=1$. $\dagger$

4. Proof of Lemma 2. The point $X=(x)$ given by (3) is interior to $K$. Indeed, assuming for the moment that $X$ is on the boundary of $K$, there is no loss of generality (suitable change

* E. Helly, Sitzungsberichte der Wiener Akademie, vol. 121 IIa (1912), p. 286 and pp. $288-9$.

† Indeed, the polynomial $\Phi(x)=a_{0}+a_{1} x+\cdots+a_{n} x^{n}$ necessarily has a real root if $a_{0}+a_{n}=0$, while the corresponding system

$$
\int_{-\infty}^{\infty} d \psi=1, \quad \int_{-\infty}^{\infty} x d \psi=\int_{-\infty}^{\infty} x^{2} d \psi=\cdots=\int_{-\infty}^{\infty} x^{n-1} d \psi=0, \quad \int_{-\infty}^{\infty} x^{n} d \psi=1
$$

has no monotonic solution $\psi(x)$. 
of axes) if we assume that the hyperplane $x_{1}=0$ is a plane of support of $K$, that is

$$
\phi_{1}(x) \geqq 0 \text { for } a \leqq x \leqq b \text { and } \int_{a}^{b} p(x) \phi_{1}(x) d x=0 .
$$

These last conditions, however, are in contradiction with $p(x)>0$ and $\phi_{1}(x) \neq 0$ in $[a, b]$. The sufficiency of the condition of Lemma 2 is thus established.

Let $a=0, b=1$, and let $K_{0}$ be the totality of points $X=(x)$ representable in the form (3) with $p(x)$ continuous and positive in $[0,1]$ and $\int_{0}^{1} p(x) d x=1$. The point set $K_{0}$ is obviously convex. We shall prove now that every point $P=\left(p_{1}, p_{2}, \cdots, p_{n}\right)$ of $K$ is a limit point of the point set $K_{0}$. Indeed, by Lemma 1 we may write

(15) $p_{k}=\int_{0}^{1} \phi_{k}(x) d \psi(x), \psi(x)$ monotonic, $\psi(0)=0, \psi(1)=1$,

for $k=1,2, \cdots, n$. Consider now the polynomial of degree $m(>1)$

(16) $P_{m}(x)=\frac{m}{m+2}\left\{B_{m}(\psi, x)+\frac{1}{m} x^{m}-\frac{1}{m}(1-x)^{m}+\frac{1}{m}\right\}$,

where

$$
B_{m}(\psi, x)=\sum_{\mu=0}^{m} \psi\left(\frac{\mu}{m}\right)\left(\begin{array}{l}
m \\
\mu
\end{array}\right) x^{\mu}(1-x)^{m-\mu}
$$

is the $m$ th Bernstein polynomial of $\psi(x) .^{*}$ From

$$
\begin{aligned}
& P_{m}^{\prime}(x)=\frac{m}{m+2}\left\{m \sum _ { \mu = 0 } ^ { m - 1 } \left[\psi\left(\frac{\mu+1}{m}\right)\right.\right. \\
&\left.\left.-\psi\left(\frac{\mu}{m}\right)\right]\left(\begin{array}{c}
m-1 \\
\mu
\end{array}\right) x^{\mu}(1-x)^{m-\mu-1}+x^{m-1}+(1-x)^{m-1}\right\},
\end{aligned}
$$

we see that

$$
P_{m}^{\prime}(x)>0,
$$

* S. Bernstein, Communications Charkow Mathematical Society, (2), vol. 13 (1912), pp. 1-2. 
for $0 \leqq x \leqq 1$. Moreover, (16) and (17) give

$$
P_{m}(0)=0, P_{m}(1)=1 \text {. }
$$

Finally, a fundamental convergence property of the polynomials of Bernstein shows that

$$
\lim _{m \rightarrow \infty} P_{m}(x)=\psi(x)
$$

for every value of $x$ where $\psi(x)$ is continuous. The point

$$
X^{(m)}: \quad x_{k}^{(m)}=\int_{0}^{1} \phi_{k}(x) P_{m}^{\prime}(x) d x, \quad(k=1,2, \cdots, n),
$$

on account of (18) and (19), belongs to the point set $K_{0}$; and, by (20) and Helly's theorem, we have $X^{(m)} \rightarrow P$ as $m \rightarrow \infty$.

If $Q$ is a fixed interior point of $K$, then $Q$ is also interior to a suitable polyhedron with $n+1$ vertices $P_{0}, P_{1}, \cdots, P_{n}$, all of which belong to $K$. By our last result we can find $n+1$ points $X_{0}, X_{1}, \cdots, X_{n}$ of $K_{0}$ such that $X_{i},(i=0,1, \cdots, n)$, is arbitrarily close to $P_{i}$. For such a sufficiently close approximation the point $Q$ will clearly be a point of the polyhedron $X_{0} X_{1} \cdots X_{n}$, hence, a point of $K_{0}$, since $K_{0}$ is convex. This concludes the proof of Lemma 2.

5. Proof of Lemma 3. (a) Let $\Delta$ have no common point with $K$. Let $\overline{A B}$ be the shortest distance from $\Delta$ to $K$, the point $A$ belonging to $\Delta$ and $B$ to $K$. Then $\Delta$ is perpendicular to the segment $A B$ so that the plane $\pi$, perpendicular to $A B$ at $A$, will contain the straight line $\Delta$. This plane $\pi$ has no point in common with $K$ (see Carathéodory, loc. cit. p. 198).

(b) Let $\Delta$ be a line of support of $K$. Let $I$ be a fixed interior point of $K$ and let $P$ be a common point of $\Delta$ and $K$. Join $I$ and $P$ by a line segment and take a point $P^{\prime}$ on its extension beyond the point $P$. Draw through $P^{\prime}$ the line $\Delta^{\prime}$ parallel to $\Delta$. Then $\Delta^{\prime}$ and $K$ have no common point. For if they had a common point $Q^{\prime}$, then the point of intersection $Q$ of $\Delta$ and $I Q^{\prime}$ would be an interior point of $K$, contradicting the fact that $\Delta$ is a line of support of $K$. According to (a) we can pass through $\Delta^{\prime}$ a bound $\pi^{\prime}$ of $K$. If now $P^{\prime}$ approaches $P$ by moving on the line $P^{\prime} P I$, the corresponding plane $\pi^{\prime}$ will move parallel to $\Delta$. For a countable number of suitably chosen positions of $P^{\prime}$, the correspond- 
ing plane $\pi^{\prime}$ will converge* to a limiting plane $\pi$, which necessarily contains $\Delta$, and which, as a limit of bounds $\pi^{\prime}$ of $K$, is a plane of support of $K$.

6. Conclusion. Finally, I wish to point out two facts. 1 . The assumptions that the functions (1) and (9), respectively, are linearly independent are not essential for Theorems 2,4 , and for Theorem 5, respectively. 2. In Lemma 2, the continuity of $p(x)$ can be replaced by the stronger condition that $p(x)$ be a polynomial. To show this one has to change in the proof given in $\S 4$ the definition of $K_{0}$ which now should be the set of points given by (3), where $p(x)$ is a polynomial, positive in $[a, b]$, with $\int p d x=1$. Our Theorems 1 and 3 can be changed accordingly, $p(x)$ being now a polynomial. $\dagger$

\section{HARVARD UNIVERSITY}

* See Carathéodory's proof (loc. cit. p. 198) for the existence of a plane of support of $K$ passing through a given boundary point of $K$.

$\dagger$ After this note was in type, I noticed several articles that are closely connected with it. In two articles by S. Kakeya, On some integral equations, I and II (Tôhoku Mathematical Journal, vol. 4 (1914), pp. 186-190, and Proceedings of the Tôkyô Mathematical-Physical Society, (2), vol. 8 (1915-16), pp. 83-102), the possibility of finding a solution $p(x)$ of the system (7) is discussed thoroughly, the solution $p(x)$ being subject to various more or less complicated auxiliary conditions. The conditions $p(x)>0, \int p d x=1$, which lead to my Lemma 2 above, were not considered by Kakeya. A note by M. Fujiwara, On the system of linear inequalities and linear integral inequality (Proceedings of the Imperial Academy of Japan, vol. 4 (1928), pp. 330-333) seems to contain (p. 332) a proof of Dines' Theorem 1, similar to the proof given in the present note. It does not actually cover the theorem, however, since Fujiwara derives there not the condition $p(x)>0$ of Theorem 1 , but only the weaker (necessary but not sufficient) condition $p(x) \geqq 0$. Finally, I may mention a beautiful note by W. Fenchel, Geschlossene Raumkurven mit vorgeschriebenem Tangentenbild (Jahresbericht der Deutschen Mathematiker-Vereinigung, vol. 39 (1930), pp. 183-185), where Lemma 2 is proved by a simple elementary method which requires no limiting process whatever, except, of course, ordinary integration. 